\title{
Front Matter: Volume 10392
}

, "Front Matter: Volume 10392," Proc. SPIE 10392, Hard X-Ray, Gamma-Ray, and Neutron Detector Physics XIX, 1039201 (9 January 2018); doi:

10.1117/12.2293178

SPIE Event: SPIE Optical Engineering + Applications, 2017, San Diego, California, SPIE. United States 


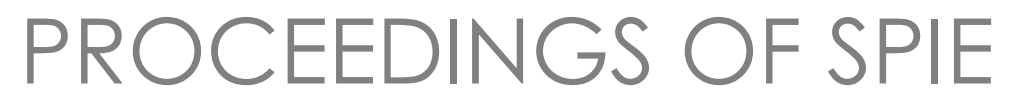

\title{
Hard X-Ray, Gamma-Ray, and Neutron Defector Physics XIX
}

\author{
Arnold Burger \\ Ralph B. James \\ Michael Fiederle \\ Larry Franks \\ Stephen A. Payne \\ Editors
}

7-9 August 2017

San Diego, California, United States

Sponsored and Published by

SPIE 
The papers in this volume were part of the technical conference cited on the cover and title page. Papers were selected and subject to review by the editors and conference program committee. Some conference presentations may not be available for publication. Additional papers and presentation recordings may be available online in the SPIE Digital Library at SPIEDigitalLibrary.org.

The papers reflect the work and thoughts of the authors and are published herein as submitted. The publisher is not responsible for the validity of the information or for any outcomes resulting from reliance thereon.

Please use the following format to cite material from these proceedings:

Author(s), "Title of Paper," in Hard X-Ray, Gamma-Ray, and Neutron Detector Physics XIX, edited by Arnold Burger, Ralph B. James, Michael Fiederle, Larry Franks, Stephen A. Payne, Proceedings of SPIE Vol. 10392 (SPIE, Bellingham, WA, 2017) Seven-digit Article CID Number.

ISSN: 0277-786X

ISSN: 1996-756X (electronic)

ISBN: 9781510612419

ISBN: 9781510612426 (electronic)

Published by

SPIE

P.O. Box 10, Bellingham, Washington $98227-0010$ USA

Telephone +1 3606763290 (Pacific Time) · Fax +1 3606471445

SPIE.org

Copyright (C) 2017, Society of Photo-Optical Instrumentation Engineers.

Copying of material in this book for internal or personal use, or for the internal or personal use of specific clients, beyond the fair use provisions granted by the U.S. Copyright Law is authorized by SPIE subject to payment of copying fees. The Transactional Reporting Service base fee for this volume is $\$ 18.00$ per article (or portion thereof), which should be paid directly to the Copyright Clearance Center (CCC), 222 Rosewood Drive, Danvers, MA 01923. Payment may also be made electronically through CCC Online at copyright.com. Other copying for republication, resale, advertising or promotion, or any form of systematic or multiple reproduction of any material in this book is prohibited except with permission in writing from the publisher. The CCC fee code is 0277-786X/17/\$18.00.

Printed in the United States of America.

Publication of record for individual papers is online in the SPIE Digital Library.

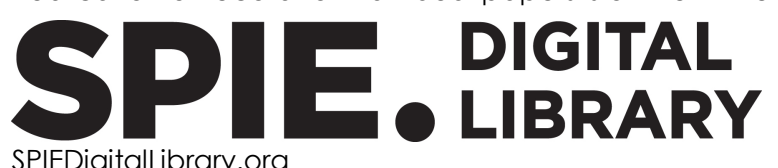

Paper Numbering: Proceedings of SPIE follow an e-First publication model. A unique citation identifier (CID) number is assigned to each article at the time of publication. Utilization of CIDs allows articles to be fully citable as soon as they are published online, and connects the same identifier to all online and print versions of the publication. SPIE uses a seven-digit CID article numbering system structured as follows:

- The first five digits correspond to the SPIE volume number.

- The last two digits indicate publication order within the volume using a Base 36 numbering system employing both numerals and letters. These two-number sets start with 00, 01, 02, 03, 04 , 05, 06, 07, 08, 09, OA, OB ... 0Z, followed by 10-1Z, 20-2Z, etc. The CID Number appears on each page of the manuscript. 


\title{
Contents
}

\author{
$\checkmark$ Authors \\ vii Conference Committee
}

\section{SCINTILLATORS I}

1039202 History and current status of strontium iodide scintillators (Invited Paper) [10392-1]

1039204 Plastic scintillator enhancement through Quantum Dot [10392-3]

1039205 Design and development of position sensitive detector for hard x-ray using SiPM and new generation scintillators [10392-4]

\section{CZT I}

1039207 Ground calibration of the spatial response and quantum efficiency of the CdZnTe hard $x$-ray detectors for NUSTAR [10392-6]

DETECTOR DEVICES

1039209 Towards the development of a SiPM-based module for the camera of the SchwarzschildCouder Telescope prototype of the Cherenkov Telescope Array [10392-8]

10392 OC A high performance neutron spectrometer for planetary hydrogen measurement [10392-11]

10392 OD A pixelated $\mathbf{x}$-ray detector for diffraction imaging at next-generation high-rate $\mathrm{FEL}$ sources [10392-12]

\section{SCINTILLATORS II}

10392 OF Directional gamma detection from the occlusion method and singular value decomposition (Invited Paper) [10392-14]

10392 OG Very fast inorganic crystal scintillators [10392-15]

$10392 \mathrm{OH}$ Development of the LunaH-Map miniature neutron spectrometer [10392-16] 
$10392 \mathrm{OL}$ High-efficiency and high-sensitivity thermal neutron detectors based on hexagonal BN epilayers [10392-20]

\section{NEUTRON DETECTORS}

10392 OM Neutron detector development for microsatellites (Invited Paper) [10392-21]

CZT III

10392 OT Mechanistic study of the accelerated crucible rotation technique applied to vertical Bridgman growth of cadmium zinc telluride (Invited Paper) [10392-28]

10392 OU Through-Silicon-Vias (TSVs) for 3D readout of ASIC for nearly gapless CdZnTe detector arrays [10392-29]

\section{OTHER GAMMA DETECTION MATERIALS}

1039210 lonizing organic compound based nanocomposites for efficient $\mathrm{Y}$-ray sensor [10392-35]

\section{DEFECT CHARACTERIZATION IN CRYSTALS}

1039212 Three-dimensional mapping and analysis of mid-gap defect distributions in TIBr by a twophoton photocurrent microscope [10392-37]

\section{POSTER SESSION}

1039214 Vertical Bridgman growth and characterization of $\mathrm{Cd}_{0.95-x} \mathrm{Mn} \mathrm{n}_{\mathrm{x}} \mathrm{Zn}_{0.05} \mathrm{Te}(\mathrm{x}=0.20,0.30)$ singlecrystal ingots [10392-39]

1039215 Improved pyroelectric x-ray generator for planetary active $x$-ray spectroscopy [10392-40]

10392 1C Challenges in the quantitative optical detection of radiation [10392-47]

10392 ID Characterization of Yb-doped silica optical fiber as real-time dosimeter [10392-48] 


\section{Authors}

Numbers in the index correspond to the last two digits of the seven-digit citation identifier (CID) article numbering system used in Proceedings of SPIE. The first five digits reflect the volume number. Base 36 numbering is employed for the last two digits and indicates the order of articles within the volume. Numbers start with 00, 01, 02, 03, 04, 05, 06, 07, 08, 09, OA, OB...0Z, followed by 10-1Z, 20-2Z, etc.

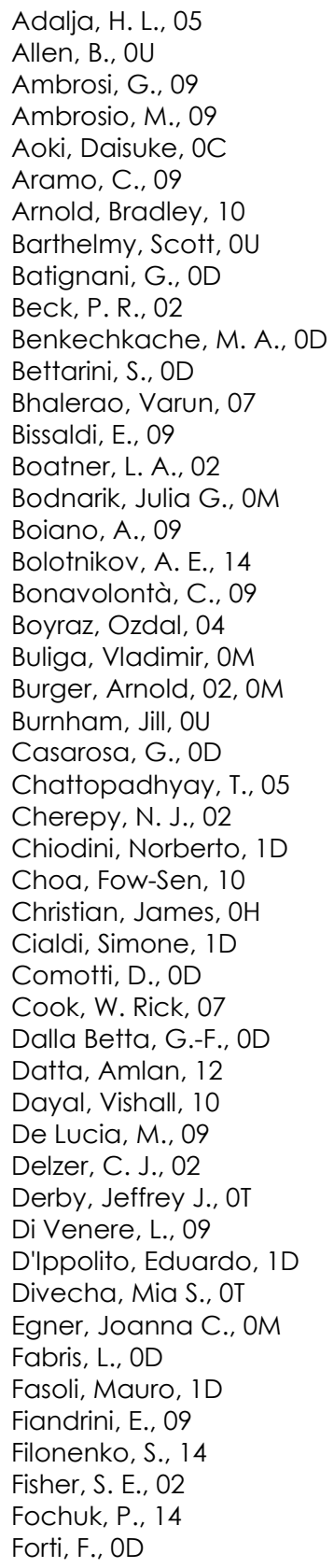

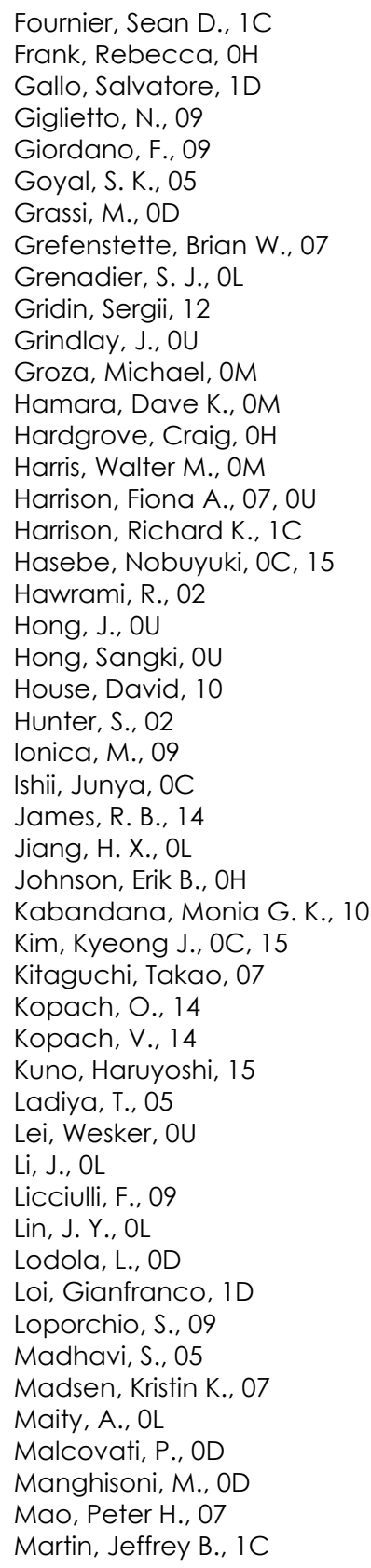

Proc. of SPIE Vol. 10392 1039201-5 
Martínez-Frías, Jesús, 0C, 15

Masone, V., 09

Matei, Liviu, OM

Matias-Lopes, José A., 0C, 15

Mendicino, R., OD

Miyasaka, Hiromasa, 07, 0U

Mizone, Miho, 15

Momayezi, M., 02

Mones, Eleonora, 1D

Morsani, F., OD

Motakef, Shariar, 12

N.P. S., Mithun, 05

Nagaoka, Hiroshi, 0C, 15

Nagrani, N., 05

Naik, Amisha P., 05

Naito, Masayuki, 0C, 15

Nilsson, Mikael, 04

Onken, Drew R., 12

Oshima, Yusuke, 15

Paladino, A., OD

Pancheri, L., OD

Paoletti, R., 09

Paoloni, E., OD

Patel, A. R., 05

Patel, V. R., 05

Patti, Robert, OU

Payne, S. A., 02

Postolache, $V_{\text {., }} 09$

Rana, Vikram, 07

Randles, M. H., 02

Ratti, L., OD

$\mathrm{Re}, V_{.,}$OD

Rizzo, G., OD

Rugliancich, A., 09

Shah, K. S., 02

Shahbazi, S., 02

Shanmugam, M., 05

Shcherbak, L., 14

Shibamura, Eido, 0C, 15

Simone, D., 09

Singh, N. B., 10

Solodovnikov, D., 02

Starr, Richard, $\mathrm{OH}$

Stassun, Keivan G., OM

Stevens, K., 02

Stoddard, Graham, $\mathrm{OH}$

Stowe, Ashley C., OM

Su, Ching-Hua, 10

Swanberg, E. L., 02

Tam, Alan, 04

Thelin, P. A., 02

Tiwari, Neeraj K., 05

Trainham, R., OF

Traversi, G., OD

Ubale, G.P., 05

Ucer, K. Burak, 12

Vacchi, C., OD

Vadawale, S. V., 05

Vagelli, V., 09

Valentino, M., 09
Vedda, Anna, 1D

Veronese, Ivan, ID

Verzellesi, G., OD

Vogel, Sam, $\mathrm{OH}$

West, Stephen, $\mathrm{OH}$

Wiemann, Dora K., 1C

Wihl, B. M., 02

Williams, Richard T., 12

$\mathrm{XU}, \mathrm{H}_{\text {., OD }}$

Zhu, Ren-Yuan, OG 


\section{Conference Committee}

Program Track Chairs

Ali M. Khounsary, Illinois Institute of Technology (United States)

Ralph B. James, Savannah River National Laboratory (United States)

Conference Chairs

Arnold Burger, Fisk University (United States)

Ralph B. James, Savannah River National Laboratory (United States)

Michael Fiederle, Freiburger Materialforschungszentrum (Germany)

Larry Franks, Consultant (United States)

Conference Co-chair

Stephen A. Payne, Lawrence Livermore National Laboratory

(United States)

Conference Program Committee

Toru Aoki, Shizuoka University (Japan)

Jim E. Baciak Jr., University of Florida (United States)

David B. Beach, Y-12 National Security Complex (United States)

Zane W. Bell, Oak Ridge National Laboratory (United States)

Koushik Biswas, Arkansas State University (United States)

Lynn A. Boatner, Oak Ridge National Laboratory (United States)

Aleksey E. Bolotnikov, Brookhaven National Laboratory (United States)

Edith Bourret Courchesne, Lawrence Berkeley National Laboratory (United States)

Mary Ellen Braunreuther, Brookhaven National Laboratory (United States)

Giuseppe S. Camarda, Brookhaven National Laboratory (United States)

Bill Cardoso, Creative Electron (United States)

Henry Chen, Brimrose Corporation of America (United States)

Nerine J. Cherepy, Lawrence Livermore National Laboratory (United States)

Jeffrey J. Derby, University of Minnesota (United States)

Kim F. Ferris, Pacific Northwest National Laboratory (United States)

Petro M. Fochuk, Yuriy Fedkovych Chernivtsi National University (Ukraine)

Jan Franc, Charles University in Prague (Czech Republic) 
Fei Gao, Pacific Northwest National Laboratory (United States)

Zhong He, University of Michigan (United States)

Keitaro Hitomi, Tohoku University (Japan)

Alan Janos, U.S. Dept. of Homeland Security (United States)

Mercouri Kanatzidis, Northwestern University (United States)

KiHyun Kim, Korea University College of Health Sciences (Korea, Republic of)

Henric Krawczynski, Washington University in St. Lovis (United States)

Kelvin G. Lynn, Washington State University (United States)

Krishna C. Mandal, University of South Carolina (United States)

Robert D. McLaren, Consultant (United States)

Shariar Motakef, CapeSym, Inc. (United States)

Sanjoy Mukhopadhyay, National Security Technologies, LLC (United States)

Madan Niraula, Nagoya Institute of Technology (Japan)

Utpal N. Roy, Brookhaven National Laboratory (United States)

Michael R. Squillante, Radiation Monitoring Devices, Inc. (United States)

Ashley C. Stowe, Y-12 National Security Complex (United States)

Csaba Szeles, Nious Technologies Inc. (United States)

Sergey E. Ulin, National Research Nuclear University MEPhl (Russian Federation)

Edgar V. van Loef, Radiation Monitoring Devices, Inc. (United States)

Aaron L. Washington II, Savannah River National Laboratory (United States)

Richard T. Williams, Wake Forest University (United States)

Kan Yang, Saint-Gobain Crystals (United States)

Session Chairs

1 Scintillators I

Stephen A. Payne, Lawrence Livermore National Laboratory (United States)

2 CZT I

Ralph B. James, Savannah River National Laboratory (United States)

3 Detector Devices

Larry Franks, Consultant (United States)

4 Scintillators II

Arnold Burger, Fisk University (United States)

5 Semiconductor Detector Materials

Aleksey E. Bolotnikov, Brookhaven National Laboratory (United States) 
6 Neutron Detectors

Arnold Burger, Fisk University (United States)

$7 \quad$ CZT II

Robert D. McLaren, Consultant (United States)

8 CZT III

Arie Ruzin, Tel Aviv University (Israel)

9 Other Gamma Detection Materials

Richard T. Williams, Wake Forest University (United States)

10 Defect Characterization in Crystals

Robert D. McLaren, Consultant (United States) 
Proc. of SPIE Vol. 10392 1039201-10 Downloaded From: https://www.spiedigitallibrary.org/conference-proceedings-of-spie on 26 Apr 2023
Terms of Use: https://www.spiedigitallibrary.org/terms-of-use 